\title{
Patrones de uso de sitios naturales y artificiales por Branta bernicla nigricans (Anseriformes: Anatidae) en Guerrero Negro, Baja California Sur, México
}

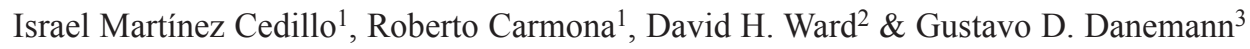 \\ 1. Departamento de Biología Marina, Universidad Autónoma de Baja California Sur, Apartado postal 19-B, La Paz \\ 23080, Baja California Sur, México; imartinez@uabcs.mx, beauty@uabcs.mx \\ 2. U. S. Geologial Survey, Alaska Science Center, Anchorage, Alaska; dward@usgs.gov \\ 3. Programa de Conservación de aves, Pronatura Noroeste A.C; gdanemann@pronatura-noroeste.org
}

Recibido 29-V-2012. Corregido 10-IX-2012. Aceptado 09-X-2012.

\begin{abstract}
Habitat use patterns of the Black Brant Branta bernicla nigricans (Anseriformes: Anatidae) in natural and artificial areas of Guerrero Negro, Baja California Sur, Mexico. The Black Brant is a common inhabitant of the Western Artic American tundra, which migrates to Southern Pacific coasts during the winter season. Approximately, 31000 birds (31\%) constitute the Mexican population of Brants at Guerrero Negro, Ojo de Liebre, and Exportadora de Sal lagoon complex; nevertheless, there is little information about the distribution patterns and zone usage. At Guerrero Negro Lagoon (GNL), Ojo de Liebre Lagoon (OLL, both natural sites), and at Exportadora de Sal (ESSA, artificial site) we determined by monthly censuses (from November 2006 to April 2007, 08:00-16:00h) and observed: (1) season and site effects on population structure (age groups), and (2) the tide level relationship with the abundance and proportion of feeding birds. Within a total of 150 observation hours and 98 birds, our results showed a general 0.68 proportion of adults, that was higher in winter than in spring. The statistics analysis showed no effects by site on the proportion of feeding birds, but we observed a temporal decrease at ESSA and at GNL. In contrast the proportion of feeding birds at OLL was constant. We observed an increase in the juveniles between winter and spring. This increase is related with the differential migration, which mentions that the juveniles are the last to leave the wintering area. In winter the relations of the tide level with the abundance of Brant were: direct at ESSA, inverse at OLL and no relation found at GNL. In spring, no relation was observed in the sites. The proportion of Brants feeding at OLL (the site with the higher abundance) was independent of the tide level. This is related with two possible behaviors of the geese: (1) they can move through the lagoon and take advantage of the tidal lag, which is up to four hours; and (2) they can modify their feeding strategies, more on floating eelgrass (Zostera marina). Rev. Biol. Trop. 61 (2): 927-935. Epub 2013 June 01.
\end{abstract}

Key words: Guerrero Negro lagoon, Brant, feeding, age groups, winter, tides.

El Ganso de collar del Pacífico (Branta bernicla nigricans Linnaeus 1758) se reproduce en la tundra alta del Ártico occidental y migra hacia el sur para pasar el invierno por las costas del Pacífico; se ha reportado que alrededor del $74 \%$ de su población (estimada en 136200 aves), inverna en el noroeste de México (Reed et al. 1998).

Antes de la década de 1960 las costas mexicanas eran poco utilizadas por el Ganso de collar, sin embargo, a partir de este período la población modificó su distribución invernal para incluir lagunas costeras más sureñas
(Reed et al. 1998). El cambio en las áreas de invernada se atribuye a la modificación de la costa oeste de los E.U.A y a la consecuente reducción de las praderas de pasto marino (Zostera marina Linnaeus 1753), su principal recurso alimenticio en invierno (Reed et al. 1998). En el noroeste mexicano la mayor parte de los gansos invernan en Bahía San Quintín (Baja California), en el complejo lagunar Guerrero Negro-Ojo de Liebre, en la Laguna San Ignacio y en Bahía Magdalena (Baja California Sur) y en el Canal Infiernillo, Sonora (Mallek \& Conant 2007). Esta especie está catalogada 
por el Gobierno Mexicano como amenazada (N.O.M. 2002).

Un factor limitante para la alimentación de esta especie en sus áreas no reproductivas es la accesibilidad a las praderas de Zostera, ya que el Ganso de collar sólo puede acceder a estas plantas durante marea baja o en aguas poco profundas (Mather et al. 1998, Reed et al. 1998, Ganter 2000, Moore et al. 2004). En diferentes zonas se ha evidenciado una relación entre el esfuerzo dedicado a la alimentación por parte del Ganso de collar y el nivel de marea (Ward et al. 2005, Moore \& Black 2006). Incluso se ha reportado que los cambios en los patrones de la marea han coincidido con los patrones de uso de las bahías por parte de los gansos (Moore \& Black 2006). De tal forma, se ha indicado que las estrategias y patrones migratorios de esta especie están modelados por gradientes latitudinales en la fenología del alimento (pasto) y en las fluctuaciones mareales (Moore et al. 2004, Ward et al. 2005, Moore \& Black 2006).
Pese a que el complejo lagunar Guerrero Negro-Ojo de Liebre-Exportadora de Sal (en adelante GN) es la zona de invernada más importante para estas aves, no existen trabajos que aborden los patrones de uso de GN por esta especie. Por lo anterior, en este estudio procuramos determinar en tres sitios clave del GN (dos naturales y uno artificial): el efecto de la época del año y el sitio en la estructura poblacional (grupos etarios), y la relación del nivel de marea con la abundancia y la proporción de aves alimentándose.

\section{MATERIALES Y MÉTODOS}

El complejo lagunar Guerrero Negro-Ojo de Liebre-ESSA se ubica en la costa centrooccidental de la Península de Baja California, dentro de la Reserva de la Biósfera del Vizcaíno (28¹3'9" - 27³4'24" N y 114²4'39" $113^{\circ} 53^{\prime} 28^{\prime}$ " W, Fig. 1). El área presenta menos de $100 \mathrm{~mm}$ de precipitación anual y una temperatura anual promedio que oscila entre los

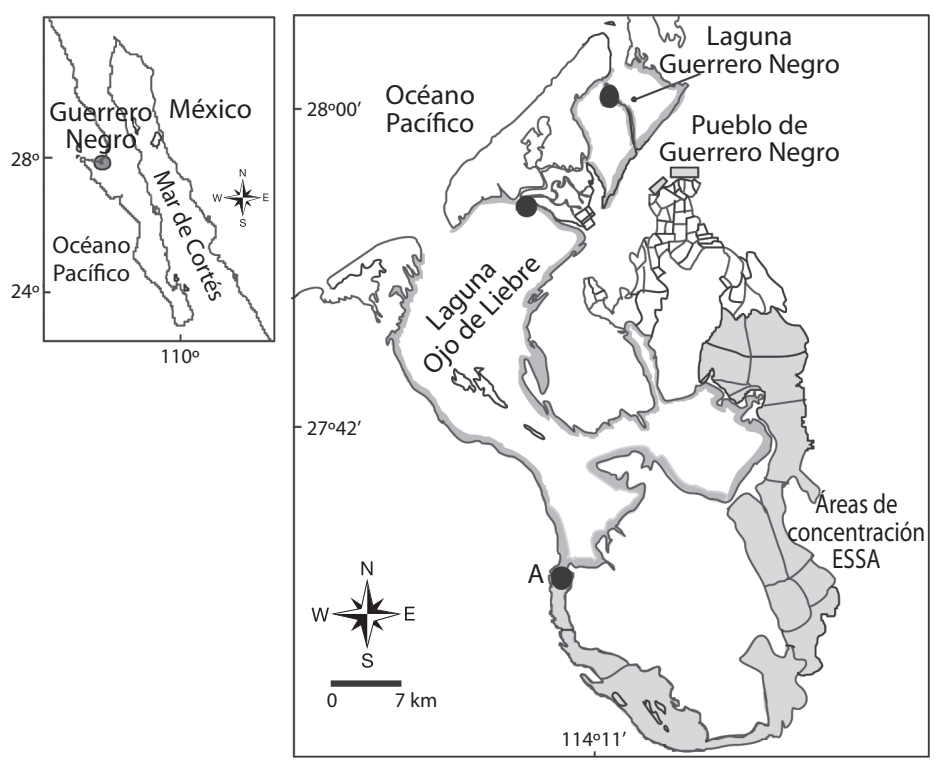

Fig. 1. Área de estudio. Se resaltan los sitios de observación: A) Salitrales 1A (ambiente artificial dentro de Exportadora de Sal), B) Puerto El Chaparrito (ambiente natural dentro de la Laguna Ojo de Liebre) y C) Camino a Puerto Viejo (ambiente natural dentro de la Laguna Guerrero Negro).

Fig. 1. Study area. Highlights the observation sites: A) Salitrales 1A (artificial environment within Exportadora de Sal), B) Puerto El Chaparrito (natural environment in Ojo de Liebre Lagoon) and C) Camino a Puerto Viejo (natural environment within the Guerrero Negro Lagoon). 
18 y $22^{\circ} \mathrm{C}$ (Contreras 1985). El complejo está formado por tres cuerpos de agua: Laguna Ojo de Liebre (LOL; $57100 \mathrm{ha}$ ), Laguna Guerrero Negro (LGN; 2100ha) y una serie de cuerpos artificiales concesionados a la Compañía Exportadora de Sal (ESSA; 33 000ha) (LluchCota et al. 1993).

De las 33000 ha de ESSA sólo 3314 son utilizadas por el Ganso de collar. Se trata de las dos zonas de acceso del agua de mar, por lo que son las más similares al ambiente natural y en ellas existen praderas de pasto marino (Carmona \& Danemann 1998). Para LOL las mayores biomasas de pasto se han registrado entre septiembre y noviembre, meses en los que también se presenta el mayor crecimiento de los brotes (Cabello-Pasini et al. 2003, CabelloPasini et al. 2004).

Como puntos de observación seleccionamos en LOL una playa adyacente al puerto

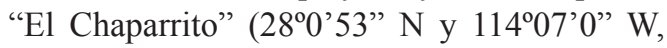
Fig. 1), la cual se caracteriza por su poca pendiente, sustrato lodoso arcilloso, con presencia de canales y de pasto; para LGN la playa

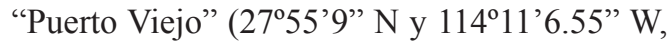
Fig. 1), también con poca pendiente y presencia de un bajo con vegetación de marisma (incluido pasto marino); para ESSA se eligió el área de concentración Salitrales 1-A (27 35' 1' N y $114^{\circ} 6$ '49" W; Fig. 1), en ésta ingresa el agua de mar al sistema, lo que favorece el establecimiento de praderas de pasto (Carmona \& Danemann 1998).

Entre noviembre de 2006 y abril de 2007 para cada sitio delimitamos $1000 \mathrm{~m}$ de línea de costa. Dentro de estos límites realizamos censos puntuales con la ayuda de binoculares (Leica 10x) y telescopios (Swarovsky 15-60x). Para cada sitio realizamos un promedio de ocho censos al mes, espaciados por una hora. Completamos cerca de $150 \mathrm{~h}$ de observación, repartidas homogéneamente entre los tres sitios; dichas observaciones se realizaron regularmente entre las 08:00 y las 16:00hs.

En cada censo determinamos: a) la abundancia (número de aves), b) actividad de éstas (alimentándose o posadas) y c) la proporción del grupo de edades: adultos e inmaduros. El criterio para diferenciar a los inmaduros de los adultos fue la presencia de bordes blancos en las plumas cobertoras en las aves inmaduras (Reed et al. 1998). Consideramos la hora del día para relacionarla con el nivel de marea (González et al. 2012).

Los análisis se realizaron con STATISTICA 5.0 (StatSoft 1995), con un nivel de significancia $(\alpha)$ de 0.05 en todos los casos. Para su análisis las variables abundancia y grupo de edad (proporción de adultos), se agruparon en dos estaciones del año: invierno (de noviembre a enero) y primavera (de febrero a abril). A continuación se describe los análisis realizados para cada caso.

Para identificar la relación entre la estructura poblacional (grupo de edad) y la estación del año y la localidad. Se formaron seis grupos de datos, esto es dos estaciones y tres sitios y se tomó como variable la proporción de aves adultas. Mediante análisis de variancia de dos vías, se probó el efecto de la temporalidad, el sitio y su interacción en la proporción de edad observada; en los casos donde se encontró una relación significativa se realizaron pruebas $a$ posteriori de Tukey (Zar 1999).

Para determinar la relación entre el nivel de marea con las abundancias y las proporciones de aves alimentándose se formaron seis grupos de datos, esto es dos estaciones del año y tres sitios, para cada grupo de información se realizó una regresión lineal simple (Zar 1999), tomando en cuenta, para todos los casos, como variable independiente el nivel de marea.

\section{RESULTADOS}

Para la determinación de los grupos de edad, en invierno se realizaron un total de 51 eventos de conteo y en primavera 47; estos incluyeron 3277 y 1827 aves, respectivamente. Es decir el grupo de edad se determinó para un total de 5104 aves, a lo largo de 98 campañas de conteo (Cuadro 1). La proporción general por grupo de edad fue de 1.47 a 1 a favor de los adultos (Cuadro 1). Dicha proporción fue afectada significativamente por la estación del año (Cuadro 1; $\mathrm{F}_{1,92}=12.7, \mathrm{p}<0.01$ ), con 
CUADRO 1

Proporción de adultos del Ganso de collar (Branta bernicla nigricans) observada por estación del año y sitio de observación. Se indica para cada caso: la proporción observada (p), su error estándar (E.e) y su tamaño de muestra (n), éste se refiere al número de conteos realizados

TABLE 1

Proportion of adult Brant (Branta bernicla nigricans) observed by season and site. It is indicated for each case: the observed proportion (p), standard error (E.e) and sample size (number of counts made, $n$ )

\begin{tabular}{|c|c|c|c|c|c|c|c|c|c|}
\hline \multirow{3}{*}{ Sitio } & \multicolumn{6}{|c|}{ Estación del año } & \multirow{2}{*}{\multicolumn{3}{|c|}{ Total }} \\
\hline & \multicolumn{3}{|c|}{ Invierno } & \multicolumn{3}{|c|}{ Primavera } & & & \\
\hline & $\mathrm{p}$ & E.e & $\mathrm{n}$ & $\mathrm{p}$ & E.e & $\mathrm{n}$ & $\mathrm{p}$ & E.e & $\mathrm{n}$ \\
\hline LOL & 0.72 & 0.06 & 16 & 0.72 & 0.7 & 12 & 0.72 & 0.05 & 28 \\
\hline LGN & 0.80 & 0.04 & 19 & 0.53 & 0.09 & 19 & 0.67 & 0.05 & 38 \\
\hline ESSA & 0.81 & 0.05 & 16 & 0.51 & 0.07 & 16 & 0.66 & 0.05 & 32 \\
\hline Total & 0.78 & 0.03 & 51 & 0.57 & 0.05 & 47 & 0.68 & 0.03 & 98 \\
\hline
\end{tabular}

una clara tendencia a disminuir entre invierno y primavera. También se pudo apreciar una mayor proporción de adultos en LOL, aunque esta relación no fue significativa (Cuadro 1; $\mathrm{F}_{2,92}=0.45, \mathrm{p}=0.63$ ). Al comparar la proporción de adultos, dividida por estación del año y el sitio, tanto en ESSA como LGN, se observó una tendencia a la disminución, aunque no significativa; en contraste, LOL fue el único lugar donde las proporciones se mantuvieron constantes a lo largo del estudio, en este último caso es resaltable el bajo valor de significancia de la prueba (Cuadro 1; $\mathrm{F}_{2,92}=2.80, \mathrm{p}=0.06$ ).

En general, las relaciones entre el nivel de marea y la proporción de aves alimentándose fueron pobres, sólo se presentaron relaciones significativas en 4 de los 12 análisis (Cuadro 2). Independientemente de su significancia, en todos los casos el modelo explicó poco de la variación observada (el $\mathrm{r}^{2}$ más alto fue de apenas 0.27 ; Cuadro 2). Con respecto a las relaciones entre el nivel de marea y las abundancias totales para el invierno se registraron tres comportamientos, en ESSA se marcó una relación directa, donde el número de aves se incrementó conforme lo hizo el nivel de marea; en contraste, en LOL, la relación fue inversa, disminuyendo el número de aves a medida que se incrementó el nivel de marea por último en LGN no hubo relación aparente (Cuadro 2).
Las pruebas estadísticas evidenciaron que sólo en LOL hubo relación significativa, explicando el modelo el $24 \%$ de la variación observada (Cuadro 2; Fig. 2). Las relaciones entre el nivel de marea y las abundancias totales para la primavera modificaron su comportamiento, en comparación al invierno, tanto en ESSA como en LOL los datos presentaron amplias dispersiones, sin tendencia aparente, mientras que en LGN se observó una relación positiva (Fig. 2). Las pruebas estadísticas corroboraron lo anteriormente descrito, pues sólo para LGN se presentó una relación significativa (Cuadro 2).

Con respecto a la relación entre la proporción del Ganso de collar alimentándose y el nivel de marea en invierno, se observaron tendencias diferentes por sitio. En ESSA la relación fue negativa, lo que indica que conforme aumentó el nivel de marea disminuyó la proporción de gansos alimentándose; contrario a este patrón en LOL se encontró una relación positiva (Fig. 2); por su parte en LGN no se observó tendencia. Los análisis estadísticos indicaron que, aunque con bajos porcentajes de variación, tanto en ESSA como en LOL hubo relaciones significativas, no así en LGN (Cuadro 2).

\section{DISCUSIÓN}

La proporción general por grupo de edad coincide con la registrada por Schamber et al. 
CUADRO 2

Regresiones lineales simples entre el nivel de mareas y la abundancia total del Ganso de collar (Branta bernicla nigricans) y la proporción de aves alimentándose; para el invierno (INV) y la primavera (PRI), en Exportadora de Sal (ESSA), Laguna Ojo de Liebre (LOL) y Laguna Guerrero Negro (LGN). Se muestra para cada caso la ordenada al origen (a), la pendiente (b), el coeficiente de determinación $\left(\mathrm{r}^{2}\right)$, los grados de libertad (g.l.), el valor del estadístico de prueba (F) y el nivel de significancia de la prueba $(\mathrm{P})$

TABLE 2

Results of simple linear regressions between tidal level and total abundance of Brant (Branta bernicla nigricans) and the proportion of birds feeding, for winter (INV) and spring (PRI), at Exportadora de Sal (ESSA), Ojo de Liebre Lagoon (LOL) and Guerrero Negro Lagoon (LGN). It is shown for each case the intercept (a), slope (b), the coefficient of determination $\left(\mathrm{r}^{2}\right)$, the degrees of freedom (g.1.), the value of the test statistic (F) and the level of significance of the test $(\mathrm{P})$

\begin{tabular}{lccccccccc} 
& Estación & Sitio & $\mathrm{a}$ & $\mathrm{b}$ & $\mathrm{r}^{2}$ & $\mathrm{~g} .1$. & $\mathrm{F}$ & $\mathrm{P}$ \\
Abundancia total & INV & ESSA & 1004.10 & 852.69 & 0.11 & 1.20 & 2.57 & 0.12 \\
& & LOL & 963.40 & 125.16 & 0.24 & 1.22 & 7.18 & $0.01^{*}$ \\
& & LGN & 678.00 & 50.93 & $<0.01$ & 1.21 & 0.01 & 0.75 \\
& \multirow{2}{*}{ MP } & ESSA & 81.79 & -3.72 & $<0.01$ & 1.23 & 0.02 & 0.89 \\
Proporción de aves alimentándose & & LOL & 478.66 & 141.14 & 0.03 & 1.24 & 0.80 & 0.38 \\
& & LGN & 497.50 & 441.25 & 0.27 & 1.24 & 9.15 & $<0.01^{*}$ \\
& & & ESSA & 55.77 & -39.37 & 0.17 & 1.20 & 4.38 & $0.05^{*}$ \\
& & LOL & 68.28 & 38.07 & 0.17 & 1.22 & 4.70 & $0.04^{*}$ \\
& \multirow{2}{*}{ MP } & LGN & 70.48 & -3.53 & $<0.01$ & 1.21 & 0.05 & 0.83 \\
& & ESSA & 36.61 & 0.79 & $<0.01$ & 1.23 & $<0.01$ & 0.96 \\
& & LOL & 57.75 & -7.91 & $<0.01$ & 1.24 & 0.24 & 0.62 \\
\hline
\end{tabular}

* =Valores significativos.

(2007), quienes indican un $32 \%$ de juveniles para esta laguna, similar al de San Quintín (31\%), pero notoriamente superior al $11 \%$ registrado para San Ignacio. Estos resultados son atribuidos a la abundancia y disponibilidad de pasto y a las interacciones sociales entre grupos familiares; pues la disponibilidad de alimento provoca una mayor cohesión familiar (Schamber et al. 2007).

La notoria disminución en la proporción de aves adultas en primavera presumiblemente se relaciona con los movimientos migratorios de esta especie (Reed et al. 1998, Poisbleau et al. 2006, Schamber et al. 2007), pues se ha registrado que la partida de las zonas de invernada se produce de acuerdo a un estatus de dominancia social, donde los primeros en salir son los grupos familiares, seguidos de las parejas con juveniles, los adultos (machos y hembras en ese orden) y por último las aves inmaduras (Poisbleau et al. 2006). Dado que las aves inmaduras no se reproducirán en el verano inmediato a su primera migración al norte, éstas pueden partir posteriormente, lo que además disminuiría la competencia intraespecífica en los sitios de paso, lo que permite explicar el aumento primaveral de las aves inmaduras en GN.

Por otra parte, la mayor proporción de adultos en LOL, la cual se mantuvo constante durante todo el período de estudio (aunque con bajos niveles de significancia), vuelve a sugerir patrones de dominancia social sobre los recursos alimenticios (Poisbleau et al. 2006). Es decir, en LOL se presentan las mayores praderas de pastos marinos (Santa María Gallegos et al. 2001, Cabello-Pasini et al. 2004), donde, como en otros sitios, es probable que predominen los grupos familiares (Moore \& Black 2006), el grupo social dominante. Así una vez empezada la migración, los grupos familiares serían los primeros en salir al norte y su lugar 

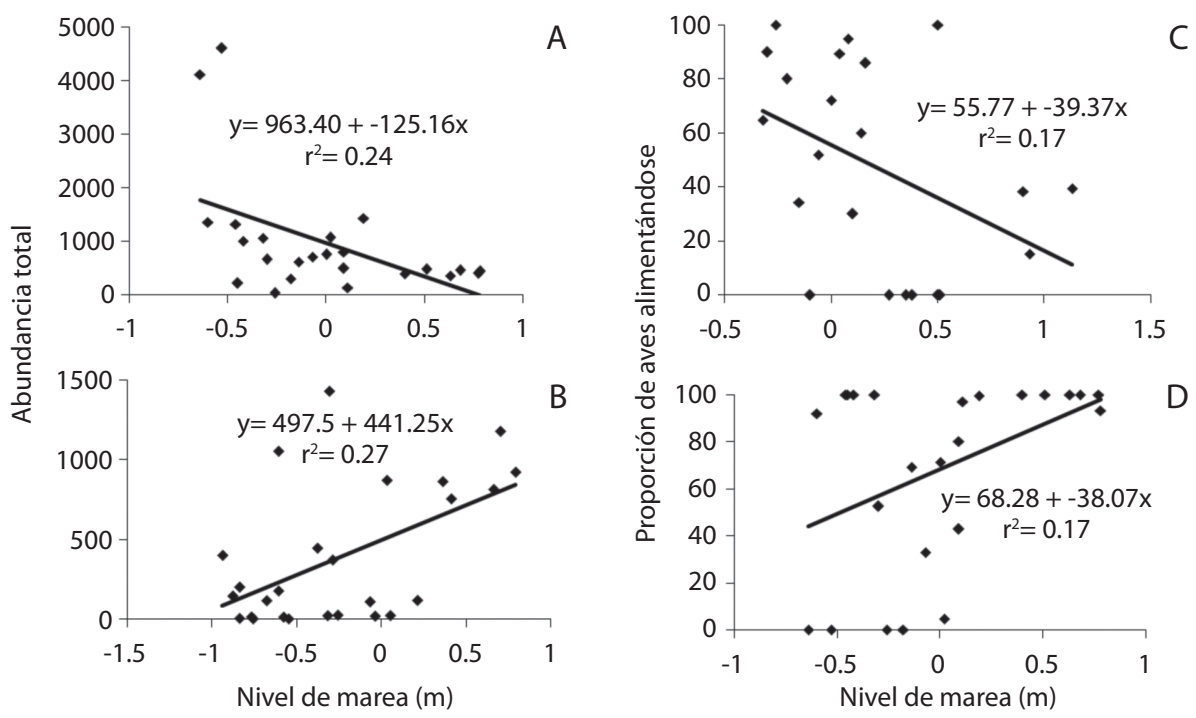

Fig. 2. Relaciones significativas entre el nivel de marea y las diferentes variables. (A) Relación entre el nivel de marea y la abundancia total del Ganso de collar, en Laguna Ojo de Liebre, para el invierno. (B) Relación entre el nivel de marea y la abundancia total del Ganso de collar, en Laguna Guerrero Negro, para la migración de primavera. (C) Relación entre el nivel de marea y la proporción de Gansos de collar alimentándose en Exportadora de Sal y (D) en Laguna Ojo de Liebre en invierno.

Fig. 2. Significant relationships between the tidal level and the different variables. (A) Relation between the tidal level and the total abundance of Brant, in Ojo de Liebre Lagoon, for the winter. (B) Relationship between the tidal level and the total abundance of Brant, in Guerrero Negro Lagoon, for the spring migration. (C) Relationship between tidal level and the proportion of feeding Brant in Exportadora de Sal and (D) in Ojo de Liebre Lagoon in winter.

sería ocupado por las aves de los grupos sociales siguientes (adultos), lo anterior permitiría explicar porqué la proporción de adultos se mantuvo constante, a diferencia de ESSA y LGN donde dichas proporciones disminuyeron, presumiblemente por los movimientos efectuados por las aves hacia zonas de mayor calidad (como LOL). Esta idea es apoyada por los resultados de Schamber et al. (2007), quienes observaron una mayor proporción de adultos en LOL en comparación con Bahía San Quintín y Laguna San Ignacio.

Efecto nivel de marea: Es de resaltar que en otros sitios como la Bahía de Humboldt se ha indicado la estrecha relación existente entre las abundancias de estas aves y el nivel de marea (Moore \& Black 2006), en contraste en GN esta relación fue poco notoria, lo que pudiera explicarse por las diferencias en las amplitudes de marea, pues en Guerrero Negro éstas son de menor magnitud en comparación con las de las zonas norteñas (Brown et al.
1989). A lo que habría que añadir la fenología del pasto marino en GN, el cual al desprenderse (noviembre; Cabello-Pasini et al. 2003), evita presumiblemente que las mareas limiten la disponibilidad del recurso. Aunque no fue un objetivo de este estudio, constantemente se observó al Ganso de collar alimentándose de pasto desprendido, tanto del varado en la línea de costa (preferentemente en marea baja) como del flotante en aguas más profundas, esta conducta presumiblemente permite que, mediante cambios en las estrategias de alimentación, el Ganso de collar responda a las modificaciones en la accesibilidad del pasto ocasionadas por el nivel de marea.

Con respecto a la relación inversa entre la abundancia de aves y el nivel de marea observada en invierno en LOL, ésta podría explicarse por el desplazamiento de los individuos desde la bocana hacia el interior de la laguna, a medida que sube la marea, buscando nuevos sitios de alimentación. La hipótesis anterior 
se sustenta en el notorio desfase (de hasta tres horas) en los niveles de mareas presentes en la bocana y los de la parte interior de la laguna.

Adicionalmente, la ausencia de relación en ESSA (área no afectada por la marea) y LGN, que por su menor tamaño presenta un desfase más reducido (menos de una hora), apoya la idea anterior. Estos movimientos permitirían explicar, al menos en parte, la baja relación entre el nivel de mareas y el comportamiento alimenticio del Ganso de collar en GN, la cual contrasta con la determinada para lugares más norteños de su ruta migratoria (Moore \& Black 2006).

Es decir, el Ganso de collar en LOL (el sitio de mayor abundancia; Martínez 2010) podría lograr la aparente independencia alimenticia respecto al nivel de marea, mediante dos estrategias no excluyentes: (1) moviéndose a lo largo de la laguna, para aprovechar el desfase en el nivel de marea y/o (2) modificando sus técnicas de alimentación, para consumir el pasto desprendido varado y flotante, esta última conducta ya ha sido registrada en otros sitios (Mather et al. 1998).

La relación positiva entre la abundancia del Ganso de collar y el nivel de marea vista en LGN en primavera puede deberse a que la zona, además de servir de área de forrajeo, es utilizada como sitio de descanso, similar a lo indicado en otras localidades (Burger et. al. 1983, Moore \& Black 2006), lo que se reflejó en que conforme aumentó el nivel de marea también lo hicieran las abundancias, razonamiento que se refuerza porque a la par del aumento en abundancias se detectó una disminución en la proporción de aves alimentándose, para las dos estaciones del año.

En el área de estudio el Ganso de collar fue constantemente observado alimentándose mediante tres diferentes estrategias: 1) ramoneando el pasto sumergido, 2) picoteando el pasto flotante en la columna de agua y 3) alimentándose del pasto varado en la playa, conductas que ya han sido descritas por otros autores (Einarsen 1965, Mather et al. 1998, Reed et al. 1998). Mather et al. (1998) que determinaron que el tipo de alimentación es dependiente del nivel de marea; los gansos prefirieron ramonear el pasto sumergido que alimentarse del pasto flotante, o del varado en la playa. Es probable que en GN las dos últimas conductas sean de mayor importancia que en otros sitios, por ejemplo, la Bahía de Humboldt, donde hasta un $90 \%$ del pasto marino queda expuesto durante la bajamar, momento que coincide con los apogeos de utilización por parte del Ganso de collar (Wilson \& Atkinson 1995, Moore \& Black 2006). Tales conductas alternativas se ven aparentemente favorecidas en GN por el desprendimiento de los pastos (Cabello-Pasini et al. 2003) y presumiblemente, ocasionan una baja relación entre el nivel de marea y la abundancia de aves alimentándose encontrada en el trabajo presente.

Por otra parte, las relaciones significativas entre el nivel de mareas y la proporción de gansos alimentándose en inverno en ESSA (negativa) y LOL (positiva) indican que ESSA funcionó más como un sitio de descanso, similarmente a lo indicado para otros ambientes artificiales (Burger et. al. 1983), pues conforme aumentó el nivel de marea, si bien se incrementó la abundancia, disminuyó la proporción de aves alimentándose. En contraste, en LOL conforme la marea aumentó disminuyó la abundancia del Ganso de collar, pero aumentó la proporción de aves alimentándose, esto probablemente se relacione con la fidelidad a las áreas de alimentación lo que permite sugerir la existencia de exclusión competitiva en los, aparentemente, mejores sitios de alimentación, tal como ha sido registrado para otras zonas (Schamber et al. 2007). El uso de ESSA, circunscrito a inicios del invierno, probablemente se relacione con la necesidad de las aves de alimentarse con mayor constancia después de la última etapa de su vuelo migratorio (Burger et al. 1983, Reed et al. 1998, Ward et al. 2005).

Recurrentemente se ha observado que el Ganso de collar abandona sus áreas de invernada cuando la disponibilidad de alimento no puede satisfacer su demanda energética (Percival \& Evans 1996, Mather et al. 1998, Reed et al. 1998, Ganter 2000, Moore et al. 2004, Ward et al. 2005, Moore \& Black 2006, Shamber et 
al. 2007). Lo anterior permite recalcar que para entender la forma en que la especie de interés utiliza las lagunas peninsulares, es imprescindible llevar a cabo estudios sobre fenología comparada de los pastos en las cuatro grandes lagunas de la zona (San Quintín, Guerrero Negro, San Ignacio y Bahía Magdalena).

En suma, en GN se observó una partida temprana de adultos en primavera. El efecto del nivel de marea fue más acusado en LGN que en LOL, presumiblemente por el tamaño de esta última (desfase de mareas) y por la presencia de una alta biomasa de pasto flotante, lo que le permite a la especie una mayor independencia alimenticia respecto al nivel de marea, por comparación con otros sitios de su ruta migratoria.

\section{AGRADECIMIENTOS}

A la compañía Exportadora de Sal S.A de C.V., por el apoyo brindado al presente trabajo, especialmente al personal de ecología (Martín García, Ramiro Zaragoza y Fabián Castillo), a Antonio Gutiérrez Aguilar, por su disponibilidad y ayuda en campo y a los jefes: Edmundo Elorduy y Martín Domínguez, por su interés en la conservación. Al personal del laboratorio de aves de la UABCS por su participación en el trabajo de campo. A Pronatura Noroeste A.C., pues este trabajo se enmarcó dentro del proyecto "Conservation of the Brant (Branta bernicla) and other migratory waterbirds in the Guerrero Negro-Ojo de Liebre coastal lagoon complex, Baja California Sur, México". A la División de Conservación de Hábitat de Aves del Servicio de Pesca y Vida Silvestre de los Estados Unidos de América, que a través del Acta para la Conservación de los Humedales de Norteamérica (NAWCA), financió parcialmente este proyecto, y en especial a Ellen C. Murphy, Coordinadora del Programa México.

\section{RESUMEN}

En el complejo lagunar Guerrero Negro-Ojo de Liebre-Exportadora de Sal inverna el 31\% de la población de Ganso de collar (Branta bernicla nigricans) que utiliza
México (31000 aves en promedio). Nosotros realizamos censos de punto mensuales (noviembre 2006 a abril 2007) en tres sitios para determinar: (1) los efectos de la época del año y del sitio en la estructura poblacional (grupos de edad) y (2) la relación del nivel de marea con la abundancia y la proporción de aves alimentándose. La proporción general de adultos fue de 0.68 . Esta proporción fue mayor en invierno que en primavera. Por sitio en LGN y ESSA disminuyó y en LOL se mantuvo constante. El incremento de aves inmaduras entre el invierno y la primavera se relaciona con su partida primaveral tardía. La alimentación del Ganso de collar en LOL (el sitio de mayor abundancia) fue independiente del nivel de marea, lo que se puede relacionar con dos conductas: (1) que las aves se muevan a lo largo de la laguna, para aprovechar el desfase, de hasta cuatro horas en el nivel de marea o (2) que modifiquen sus estrategias de alimentación, para consumir el pasto flotante.

Palabras claves: Laguna Guerrero Negro, Ganso de collar, alimentación, invernada, mareas.

\section{REFERENCIAS}

Brown, J., A. Colling, D. Park, J. Phillips, D. Rothery \& J. Wright. 1989. Waves, tides and shallow-water processes. In G. Berman (eds.). The Open University, Inglaterra.

Burger, J., R. Trout, W. Wander \& G. Ritter. 1983. Jamaica Bay studies: IV. Abiotic factors affecting abundance of brant and Canada geese on an east coast estuary. Wilson Bull. 95: 384-403.

Carmona, R. \& G. Danemann. 1998. Distribución espaciotemporal de aves en la salina de Guerrero Negro, Baja California Sur, México. Cienc. Mar. 24: 389-408.

Cabello-Pasini, A., R. Muñiz-Salazar \& D. Ward. 2003. Annual variations of biomass and photosynthesis in Zostera marina at its end of distribution in the North Pacific. Aquat. Bot. 76: 31-47.

Cabello-Pasini, A., R. Muñiz-Salazar \& D. Ward. 2004. Caracterización bioquímica del pasto Zostera mari$n a$ en el límite sur de su distribución en el Pacífico Norte. Cienc. Mar. 30: 21-34.

Contreras, F. 1985. Las lagunas costeras mexicanas. Centro de Ecodesarrollo. Secretaría de Pesca, México.

Einarsen, A.S. 1965. Black brant: sea goose of the Pacific coast. University of Washington, Seattle, EE.UU.

Ganter, B. 2000. Seagrass (Zostera spp.) as food for brent geese (Branta bernicla): an overview. Helgol. Mar Res. 54: 63-70.

González, J., A. Morales \& J.L. Ochoa 2012. Predicción de mareas, Centro de Investigación Científica y Educación Superior de Ensenada, Ensenada, Baja California, México (Consultado: 1 mayo 2009, http:// oceanografia.cicese.mx/predmar). 
Lluch-Cota, D.B., A. Castellanos-Vera, J. Llinas-Gutiérrez \& A. Ortega-Rubio. 1993. La Reserva de la Biósfera del Vizcaino, p. 358-388. In S. Salazar-Vallejo \& N.E.N. Gonzáles (eds.). Biodiversidad Marina y Costera de México. CONABIO-CIQRO, México, D.F., México.

Mallek, E. \& B. Conant. 2007. Winter waterfowl survey. México west coast and Baja California. January 2007. U.S. Fish and Wildlife Service.

Martínez, I. 2010. Distribución y abundancia del Ganso de collar (Branta bernicla nigricans) en el complejo lagunar guerrero Negro-Ojo de Liebre-ESSA, México. Tesis de Maestría, Universidad Autónoma de Baja California Sur, La Paz, México.

Mather, R.G., W.I. Montgomery \& A.A. Portig. 1998. Exploitation of intertidal Zostera species by brent geese (Branta bernigla hrota): why dig for your dinner? Biol. Environ. 3: 147-152.

Moore, J.E., M.A. Colwell, R.L. Mathis \& J.M. Black. 2004. Staging of Pacific flyway brant in relation to eelgrass abundance and site isolation, with special consideration of Humboldt Bay, California. Biol. Conserv. 115: 475-486.

Moore, J.E. \& J.M. Black. 2006. Slave to tides: spatiotemporal foraging dynamics of spring staging black brant. Condor 108: 661-677.

NOM-059-ECOL-2001. Protección ambiental, que determina las especies y subespecies de flora y fauna silvestre terrestre y acuática en peligro de extinción, amenazadas y las sujetas a protección especial, y que establece especificaciones para su protección. Diario Oficial de la Federación 16 de marzo de 2002. México, D.F., México.

Percival, S.M. \& P.R. Evans. 1996. Brent Geese Branta bernicla and Zostera; factors affecting the exploitation of a seasonally declining food resource. Ibis 139: 121-128.

Poisbleau, M., H. Fritz, M. Valeix, P.Y. Perroi, S. Dalloyau \& M.M. Lambrechts. 2006. Social dominance correlates and family status in wintering dark-bellied brent geese, Branta bernicla bernicla. Anim. Behav. 71: 1351-1358.

Reed, A., D.H. Ward, D.V. Derksen \& J.S. Sedinger. 1998. Brant (Branta bernicla). In The Birds of North America, No. 337. A. Poole \& F. Gill (eds.). The Birds of North America, Inc., Filadelfia, PA.

Santa María Gallegos, N.A., R. Riosmena Rodríguez \& J.L. Sánchez Lizaso. 2001. Las praderas de Zostera marina L. en la Reserva de la Biosfera El Vizcaíno, México. Actas de las I Jornadas sobre Reservas Marinas. Murcia, España, marzo de 1999. Ministerio de Agricultura, Pesca y Alimentación, España.

Schamber, J.L., J.S. Sedinger, D.H. Ward \& K.R. Hagmeier. 2007. Latitudinal variation in population structure of wintering Pacific Black Brant. J. Field Ornithol. 78: 74-82.

StatSoft. 1995. Statistica for Windows version 5.0 StatSoft Inc. Tulsa, Oklahoma, EE.UU.

Ward, D.H., A. Reed, J.S. Sedinger, J.M. Black, D.V. Derksen \& P. Castelli. 2005. North American Brant: effects of changes in habitat and climate on population dynamic. Global Change Biol. 11: 869-880.

Wilson, U.W. \& J.B. Atkinson. 1995. Black brant winter and spring-staging use at two Washington coastal areas in relation to eelgrass abundance. Condor 97: 91-98.

Zar, J.H. 1999. Biostatistical analysis. Prentice Hall, Nueva Jersey, EE.UU. 
